\title{
SOME OBSERVATIONS OF 3I SPINAL CORD INJURY PATIENTS ON WHOM THE BRICKER PROCEDURE WAS PERFORMED
}

\author{
By Benjamin A. Moeller, JR., B.S., M.D., F.A.C.P. \\ Spinal Cord Injury Service, Veterans Administration Hospital, Memphis, Tennessee, U.S.A.
}

Abstract. Only one-half of 3I spinal cord injury patients had good to fair results following cutaneous uretero-ileostomy. Poor results and deaths with urinary complications were more prevalent in the cervical and upper thoracic than in the more caudad cord lesions. There was a spread of $\frac{1}{2}$ to I9 years in time interval from onset of spinal cord injury to performance of the Bricker procedure. A tendency for unsatisfactory postoperative courses was more associated with the longer time intervals.

Key words: Bricker procedure; Contra-indication; Uretero-ileostomy.

\section{Introduction}

THE question: How fare spinal cord injury individuals with the Bricker procedure?

Bricker (1950) reported on four patients with cutaneous uretero-ileostomy after pelvic evisceration.

He listed the operative features that produced good results (Bricker, I956). The essential advantages of the ileal segment is that active peristalsis tends to keep the segment empty when brought to the skin surface so that it acts as a conduit rather than a reservoir, reducing stasis and subsequent ascending infection and electrolyte reabsorption (MacKenzie \& Ankenman, 1960).

The history of use of isolated segments of small intestine for the urinary tract, appears in DeWeerd's paper (1959).

Comarr (1972) has shown that patients with Schneider's central cord syndrome of cervical injury can have normal return of bladder function as long as 8 months after injury. Insoft et al. (I97I) suggest that diversion be postponed for at least I year to allow an adequate period for recovery.

Watt (1974) has a lucid article on nursing care for urinary diversions.

The routine irrigation of the infected bladder with antiseptic solutions has prevented the development of early pyocystitis (Kambouris et al., I976).

Cordonnier and Bowles (1970) reported on 60 cases of ileal loop diversion for neurogenic bladder. They thought it was especially applicable in traumatic paraplegia when progressive upper urinary tract deterioration is occurring. Their total of 436 ileal loops for many conditions were followed up to I 5 years.

Kambouris et al. (I976) reported on 26 patients with spinal cord injury followed up to 6 years after the Bricker procedure.

Woodhead and Porch (1964) followed patients an average of 20 months from the time of surgery. They remarked that it was beginning to appear that by judicious use of prophylactic cutaneous uretero-ileostomy on those who have not yet sustained significant urinary tract damage, such damage can be largely prevented.

A favourable report on II Bricker procedures on paraplegics came out of Richmond VA Hospital in I965 (Hackler et al., 1965). Kenealy (1965) in discussing this paper noted that once anatomic scarring occurs in the collecting 
system and renal parenchyma, stasis tends to be perpetuated. Ten years later Reece and Hackler (1975), at Richmond, reported 3 to 5 year poor results with three ileal conduits following successful bilateral vesico-ureteroplasty.

Thirty-five per cent of Bors and Comarr (I97I) observed 20 cases of cutaneous uretero-ileostomy died. Comarr (I97I, I972), for up to I3 years after Bricker procedure, followed i I cases performed at hospitals elsewhere.

Guttmann (I973) commented on unfavourable results of ileal conduits urinary diversion.

Duggan et al. (1974) showed the disadvantages of reflux in uretero-ileal cutaneous anastomosis. Skinner and others (1975) have demonstrated that ureteral reflux from ileal conduits produces histologic evidence of pyelonephritis.

At Memphis we didn't think the uretero-ileal anastomosis was adequate unless reflux occurred on ileogram!

Comarr and Bors (I97I, I972) point out the desired ability to performed retrograde catheterisation being negated by the Bricker procedure.

\section{Materials}

I reviewed the available records and X-ray films to find out what happened to spinal cord injury individuals after the Bricker procedure.

A markedly incomplete central cord syndrome and all individuals with known neoplasm were excluded from the study. This left 3I patients who were followed an average of 4.8 years after cutaneous uretero-ileostomy. Fourteen of the 3 I followed 5 to I 5 years. Twenty-two of these patients had their ureteroileostomies performed at elsewhere hospitals, nine at Veterans Administration Hospital, Memphis.

The nine Memphis procedures were performed in adult paraplegics for similar reasons to Bors and Comarr (I97I) observed 20 cases. These were: recurrent urinary infection (Flickinger \& Walker, I958), with and without reflux, hydronephrosis and/or deterioration of renal function.

\section{Method}

The spinal cord region involved in traumatic myelopathy, age of patient at time of injury and at time of Bricker procedure and number of years known alive with a Bricker bladder were tabulated. The pertinent aspects of urinary tract status were used to classify results into good, fair, poor and death categories.

\begin{tabular}{|c|c|c|c|}
\hline & & & esults \\
\hline & & $\mathrm{C}_{4}-\mathrm{C}$ & 3 MYELOPATHY \\
\hline $\begin{array}{l}\text { Patient and } \\
\text { age injury }\end{array}$ & $\begin{array}{l}\text { Age at } \\
\text { Bricker }\end{array}$ & $\begin{array}{c}\text { Alive } \\
\text { W Bricker }\end{array}$ & Remarks \\
\hline & & per Motor I & Neuron (UMN) Bladder \\
\hline & & & ood Results \\
\hline $\begin{array}{l}\text { No. I4 H. R. } \\
24 \mathrm{yr}\end{array}$ & $35 \mathrm{yr}$ & $7 \mathrm{yr}$ & $\begin{array}{l}\text { Bilateral hydroureters and hydronephrosis cleared on } \\
6 \text { yr post-Bricker IVP }\end{array}$ \\
\hline $\begin{array}{l}\text { No. } 26 \text { A. C. W. } \\
36 \mathrm{yr}\end{array}$ & $43 \mathrm{yr}$ & $3 \mathrm{yr}$ & I yr post-Bricker IVP and renal function normal \\
\hline $\begin{array}{l}\text { No. } 30 \text { A. K. W. } \\
24 \text { yr }\end{array}$ & $29 \mathrm{yr}$ & I I yr & $\begin{array}{l}\text { I I yr post-Bricker caliectasis left upper kidney pole. } \\
\text { Creatinine clearance normal }\end{array}$ \\
\hline
\end{tabular}




\section{Patient and} age/injury

\begin{tabular}{|c|c|}
\hline $\begin{array}{l}\text { No. } 29 \text { T. J. M. } \\
20 \mathrm{yr}\end{array}$ & $20 \mathrm{yr}$ \\
\hline $\begin{array}{l}\text { No. Io W. L. C. } \\
32 \mathrm{yr}\end{array}$ & $42 \mathrm{yr}$ \\
\hline $\begin{array}{l}\text { No. } 5 \text { F. N. G. } \\
24 \text { yr }\end{array}$ & $26 \mathrm{yr}$ \\
\hline $\begin{array}{l}\text { No. } 4 \mathrm{~J} . \mathrm{yr} \\
35 \mathrm{~F} .\end{array}$ & $38 \mathrm{yr}$ \\
\hline $\begin{array}{l}\text { No. } 2 \text { W. H. B. } \\
45 \text { yr }\end{array}$ & 5I yr \\
\hline $\begin{array}{l}\text { No. } 8 \mathrm{~B} \text {. L. M. } \\
\text { M. }\end{array}$ & $39 \mathrm{yr}$ \\
\hline
\end{tabular}

No. I6 J. B. B. $\quad 26 \mathrm{yr}$ $2 \mathrm{I} \mathrm{yr}$ No. 34 C. L. B. $\quad 49 \mathrm{yr}$

No. I2 F. W. C. $44 \mathrm{yr}$ $4 \mathrm{I} \mathrm{yr}$

No. 20 E. M. $34 \mathrm{yr}$

$37 \mathrm{yr}$

No. 15 I. A. H. $37 \mathrm{yr}$

$44 \mathrm{yr}$

No. 18 W. P. F. $50 \mathrm{yr}$

No. 3I W. E. N. $46 \mathrm{yr}$ $28 \mathrm{yr}$

No. I J. E. B. $26 \mathrm{yr}$

No. 25 D. T. T. $\quad 4 \mathrm{I}$ yr $26 \mathrm{yr}$
Alive

W/Bricker

Remarks

Fair Results

I yr I yr post-Bricker ileo loopgram reflux only right ureter. IVP normal

$3 \mathrm{yr}$ Hypertension $3 \mathrm{yr}$ pre-Bricker I yr post-Bricker cerebrovascular accident

Poor Results

5 yr Postoperative temp $107^{\circ}$ brain damage. IVP prior and 3 yr post-Bricker normal

$4 \mathrm{yr} 2 \mathrm{y} r$ pre-Bricker right pyelolithotomy. I 2 yr postBricker right nephrectomy

perative Lower Motor Neuron (LMN) Bladder

Poor Results

$3 \mathrm{yr}$ Prior to Bricker bilateral ureteral reflux to pelves. 2 yr post-Bricker right pelviolithotomy

$4 \mathrm{yr} \quad$ I 3 yr post-Bricker stenosed left distal ureter revised. $2 / 3 \mathrm{yr}$ post-Bricker creatinine clearance $60 \mathrm{cc} / \mathrm{min}$. (I $26 \mathrm{cc} / \mathrm{min}$ pre-Bricker)

T4 Myelopathy

UMN Bladder

Good Results

2 yr Pre-Bricker IVP normal. I yr post-Bricker mild right ureteropyeloectasis. No obstruction on ileoconduitgram. $24 \mathrm{hr}$ creatinine clearance normal pre and $2 \mathrm{yr}$ post-Bricker

T7-Ti2 Myelopathy

UMN Bladder

Good Results

$2 \mathrm{yr} \quad$ IVP normal $2 \mathrm{yr}$ post-Bricker

$3 \mathrm{yr}$ Infusion IVP prior Bricker bilateral pyelocaliectasis. I $\mathrm{yr}$ post-Bricker IVP normal. $2 \mathrm{yr}$ post-Bricker blood creatinine normal

Fair Results

IO yr Post-Bricker: $5 \mathrm{yr}$-stenotic left ureter, ileo site reanastomosed. $6 \mathrm{yr}$-conduitgram: bilateral caliectasis. Io $\mathrm{yr}-24 \mathrm{hr}$ creatinine clearance I I $7 \mathrm{cc} / \mathrm{min}$

Poor Results

$5 \mathrm{yr} \quad 3 \mathrm{yr}$ post-Bricker right pelviolithotomy and nephrostomy. $5 \mathrm{yr}$ post-Bricker right uretero-ileostenosis. IVP decrease function left kidney. Creatinine clearance $77 \mathrm{cc} / \mathrm{min}$. (pre-Bricker $58 \mathrm{cc} / \mathrm{min}$ )

LMN Bladder

Good Results

3 yr IVP normal I yr post-Bricker

$5 \mathrm{yr}$ Pre and $4 \mathrm{yr}$ post-Bricker IVP. Grade I hydronephrosis

$4 \mathrm{yr}$ post-Bricker $24 \mathrm{hr}$ creatinine clearance $99 \mathrm{cc} / \mathrm{min}$

Poor Results

$7 \mathrm{yr} \quad 6 \mathrm{yr}$ post-Bricker non-functioning right kidney excised

$4 \mathrm{yr} \quad$ Bricker to remaining left kidney. $4 \mathrm{yr}$ post-Bricker marked renal insufficiency and hypertension 


\section{Li-CaUdad Radiculopathy}

Patient and age/injury
Age at Bricker

Alive

W/Bricker
Remarks

\section{LMN Bladder \\ Good Results}

No. I9 S. R. P.
36 yr
$\begin{aligned} & \text { No. 2 I D. M. } \\ & 38 \text { yr }\end{aligned}$

No. 22 B. J. P.

No. 7 J. B. H.

No. I 3 J. W. G. $60 \mathrm{yr}$ $55 \mathrm{yr}$ $30 \mathrm{yr}$ $33 \mathrm{yr}$

$39 \mathrm{yr}$

$35 \mathrm{yr}$ $35 \mathrm{yr}$

No. 17 B. R. E. $\quad 30 \mathrm{yr}$ $23 \mathrm{yr}$

Patient and age/injury

\section{Age at} Bricker

No. 33 R. D. P.

$44 \mathrm{yr}$

Died after
Bricker

$3 \mathrm{yr}$

$5 \mathrm{yr}$

\section{Fair Results}

I5 yr I4 yr post-Bricker IVP compatible with bilateral chronic pyelonephritis

IVP 3 yr post-Bricker hydronephrosis Grade I right, Grade II left. $24 \mathrm{hr}$ creatinine clearance normal

Poor Results

$3 \mathrm{yr} \quad 3 \mathrm{yr}$ post-Bricker bilateral pelviolithotomy

\section{C6 UMN Bladder}

3 yr Pre-Bricker BUN $20 \mathrm{mg} \%$. IVP slight blunting calices, rt. I yr post-Bricker IVP delayed function, right kidney. 2 yr post-Bricker BUN $37 \mathrm{mg} \%$. Stones from conduit prior to death by telephonic report

$5 \mathrm{yr}$ Pre-Bricker IVP normal. Bilateral reflux to pelves. $5 \mathrm{yr}$ post-Bricker non-function left kidney and $9 \mathrm{~mm}$ flat calculus. Death due thrombotic occlusion abdominal aorta and renal arteries

\section{C6 Operative LMN BLAdDER}

No. 24 A. D. S. 5 I yr $47 \mathrm{yr}$

No. 35 T. O. W. $38 \mathrm{yr}$

$45 \mathrm{yr}$

No. i I C. L. S. $\quad 38 \mathrm{yr}$ $32 \mathrm{yr}$
$3 \mathrm{yr}$

Post mortem: recent myocardial infarction, bilateral pyelonephritis and left pyohydronephrosis

\section{TI UMN BLADDER}

7 yr 3 yr pre-Bricker small right renal calculus. PreBricker reflux to calices. 2 months post-Bricker bilateral small renal calculi

\section{TII-TI2 LMN BLADDER}

$8 \mathrm{yr}$ IVP pre-Bricker bilateral reflux to dilated calices. $4 \mathrm{yr}$ post-Bricker IVP no function left, poor $20 \mathrm{~min}$. function right. Pre-Bricker BP I Io/80, 6 yr postBricker BP I84/100. Post mortem: lobular pneumonia. Severe bilateral pyelonephritis, chronic hydronephrosis, several calyceal stones, right 
Urinary complications occurred in $\mathrm{I} 4$ of the $3 \mathrm{I}$ patients with 6I renal units (see Table I). Time intervals for complications after the Bricker procedure are listed in Table II.

Herein listed are deaths and urinary complications:

\section{TABLE I}

Complications in 14 of 31 patients with 6I renal units

\begin{tabular}{lc}
\hline & Renal units \\
\hline Non-function kidney, left & 2 \\
Kidney excised, right & \\
$\quad$ I non-function and infected & \\
$\quad$ I had pre-Bricker pyelolithotomy & 2 \\
Ureteral stenosis & 3 \\
Hydronephrosis & 3 \\
Perforation ureteroileal sites & 2 \\
$\quad$ death 40 days post-Bricker & 9 \\
Renal calculi & - \\
\hline
\end{tabular}

\section{TABLE II}

Time after Bricker complications

\begin{tabular}{|c|c|c|c|c|c|}
\hline $\begin{array}{l}\text { Bricker } \\
\text { time from } \\
\text { injury }\end{array}$ & $\begin{array}{l}\text { Time } \\
\text { from } \\
\text { Bricker }\end{array}$ & No. & Right kidney & Left kidney & Deceased after Bricker \\
\hline $3 \mathrm{yr}$ & $\mathrm{I} / 2 \mathrm{yr}$ & 4 & Nephrectomy & - & $3 \mathrm{yr}$ (report of stones from conduit) \\
\hline $7 \mathrm{yr}$ & I $\mathrm{yr}$ & I7 & IVP delayed function & 一 & - \\
\hline $6 \mathrm{yr}$ & $\begin{array}{l}2 \mathrm{yr} \\
2 \mathrm{yr}\end{array}$ & $\begin{array}{r}2 \\
27\end{array}$ & $\begin{array}{l}\text { Pelviolithotomy } \\
\text { Revision uretero-ileal }\end{array}$ & 二 & $3 \mathrm{yr}$ (necrotic $\overline{\text { renal papillitis and }}$ \\
\hline $7 \mathrm{yr}$ & $2 \mathrm{yr}$ & 27 & site and ileal stoma & & $\begin{array}{l}3 \text { perirenal abscess) } \\
\text { papintis and }\end{array}$ \\
\hline $7 \mathrm{yr}$ & $3 \mathrm{yr}$ & 15 & $\begin{array}{l}\text { Pelviolithotomy and } \\
\text { Nephrostomy }\end{array}$ & - & - \\
\hline $5 \mathrm{yr}$ & $3 \mathrm{yr}$ & 13 & Pelviolithotomy & Pelviolithotomy & \\
\hline $4 \mathrm{yr}$ & $3 \mathrm{yr}$ & 24 & - & Pyohydronephrosis & $3 \mathrm{yr}$ (myocardial infarction) \\
\hline $6 \mathrm{yr}$ & $4 \mathrm{yr}$ & I I & - & No function & 8 yr (lobular pneumonia and \\
\hline $7 \mathrm{yr}$ & $5 \mathrm{yr}$ & 20 & - & $\begin{array}{l}\text { Uretero-ileal site } \\
\text { reanastomosed }\end{array}$ & \\
\hline $9 \mathrm{yr}$ & $5 \mathrm{yr}$ & 33 & - & Non-function & 5 yr (thrombotic occlusion aorta \\
\hline I I yr & $5 \frac{1}{2} \mathrm{yr}$ & 32 & $\begin{array}{l}\text { IVP no function and } \\
\text { stones }\end{array}$ & $\begin{array}{l}\text { IVP no function } \\
\text { and stones }\end{array}$ & $\begin{array}{l}6 \text { yr (bilateral renal abscess forma- } \\
\text { tion) }\end{array}$ \\
\hline I4 $\mathrm{yr}$ & $6 \mathrm{yr}$ & I & Nephrectomy & - & \\
\hline $8 \mathrm{yr}$ & days & 36 & & & $\begin{array}{l}40 \text { days (bilateral uretero-ileal } \\
\text { perforation) }\end{array}$ \\
\hline $17 \mathrm{yr}$ & 2 months & 35 & Small calculi & Small calculi & $7 \mathrm{yr}$ \\
\hline
\end{tabular}


There were three primarily urinary tract deaths:

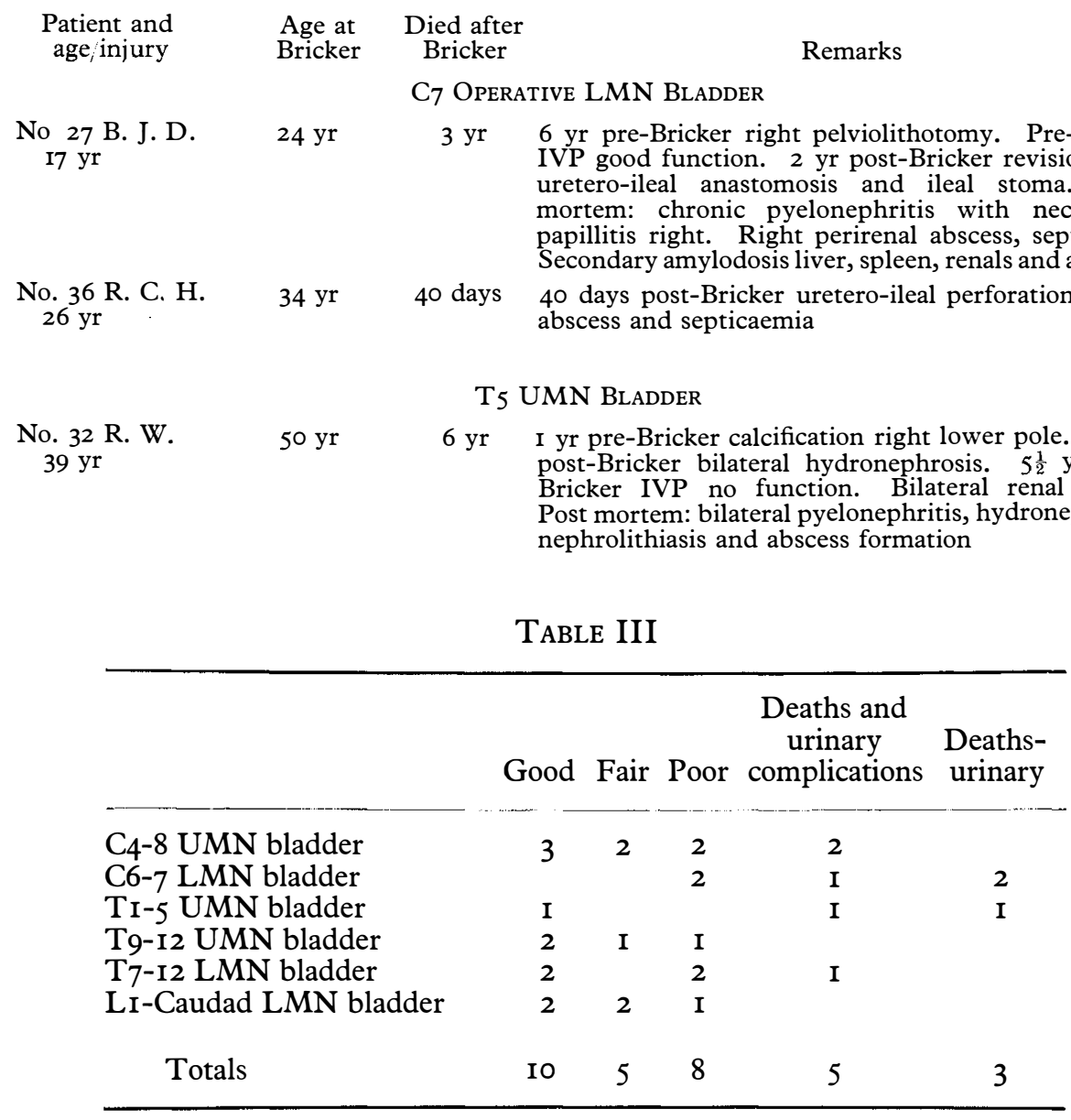

Correlation of results and dermatome levels of 3I patients with Bricker procedures is shown in Table III.

\section{Discussion}

One-half of operated patients had a satisfactory urinary tract course during review period.

Following the Bricker procedure, there were known urinary complications and deaths of:

5 of the I 4 cervical cases within a median of 3 years.

2 of the 3 upper thoracic lesions, 6 and 7 years postoperative.

I of the 9 lower thoracic lesions in 8 years.

None of five cauda equina lesions were known dead. The five averaged being alive 6.4 years after Bricker. (Range 3 to 15 years.)

Of the eight deaths, three were primarily due to urinary tract complications. 


\section{Conclusions}

The $1946-76$ known death rate of the 3790 spinal cord injury patients from Memphis Veterans Administration Hospital is 12.7 per thousand per year.

During an average of 4.8 years, 8 of $3 \mathrm{I}$ patients with cutaneous ureteroileostomy died (about 53.8 per thousand per year).

Only one-half of the patients with Bricker procedure had a fair to good result. As a therapeutic procedure for the non-neoplastic urinary tract complications of spinal cord injury, the Bricker procedure is contra-indicated.

\section{ZUSAMMENFASSUNG}

Nur die Hälfte der Patienten, die eine Bricker Operation halten, zeigten gute Resultate. Die Bricker Operation ist für nicht-neoplastische Prozesse der Brickenmarks, i.e. traumatische Läsionen kontraindiziert.

Acknowledgement. Mrs Mary A. Thannisch contributed much to format. She and Mrs Jackie M. Tombone were of much help with the references.

\section{REFERENCES}

Bors, E. \& Comarr, A. E. (I97I). Neurological Urology. Physiology of Micturation, Its Neurological Disorders and Sequelae. University Park Press, Baltimore, pp. 247-253.

BRICKeR, E. M. (I950). Bladder substitution after pelvic evisceration. Surgical Clinics of North America, 30, I 5 I I-I 52 I.

BRICKER, E. M. (I956). Substitution for the urinary bladder by the use of isolated ileal segments. Surgical Clinics of North America. 36, I I I7-I I30.

ComarR, A. E. (I97I). Follow-up on patients having undergone ileal conduit and vesicostomy operations. Proceedings of the Eighteenth Veterans Administration Spinal Cord Injury Conference. Foint Meeting with The International Medical Society of Paraplegia, pp. I 79-I 82, October 5-7.

ComArR, A. E. (I972). Renal complications of the ileal conduit and cutaneous vesicostomy among patients with traumatic cord bladders. F. Urology, 107, 762-765.

CoRdonnieR, J. J. \& Bowles, W. T. (1970). Ileal loop-urinary diversion. Surgery of the ureter and urinary conduits. In: Urology, ed. M. F. Campbell and J. H. Harrison. W. B. Saunders Company, Philadelphia, 3rd edition, Chap. 58, pp. 232 I-2337.

DeKernion, J. B. et al. (I969). Treatment of late complications of ileal conduit. $\mathcal{F}$. Urology, 102, 320-324.

DeRosa, F. P. (1958). Complications incident to the ileal conduit. F. Urology, 79, 834-837.

DeWeERd, J. H. (I959). Urinary diversion via an ileal segment. Surgical Clinics of North America, 39, 907-925.

Dicus, D. R. (1974). New perspectives in the construction of the ileal stoma. F. Urology, II2, 59I-592.

Duggan, F. J., JR, SANFord, E. J. \& Rohner, T. J., JR. (1974). The disadvantages of reflux in uretero-ileal cutaneous anastomoses for supravesical urinary diversion. Urological Research, 2, 85-90.

FlickingeR, T. L. \& WALTER, W. H. (1958). The use of the Bricker procedure in vesicalureteral reflux. Proceedings of the Seventh Annual Clinical Paraplegia Conference, p. 56-7I, November I8-20, I958. VA Medical Teaching Group Hospital, Memphis, Tn.

Gutrmann, L. (1973). Spinal Cord Injuries. Comprehensive Management and Research. Blackwell Scientific Publications, London, pp. 40I-407.

Hackler, R. H., Dalton, J. J., JR., \& BunTs, R. C. (1965). Changing concepts in the preservation of renal function in the paraplegic. F. Urology, 94, IO7-I I I.

Insoft, J., Galleher, E. P., JR. \& YounG, J. D., JR. (I97I). Urinary diversion for neurogenic bladder: use of retroperitoneal ileal loop. f. Urology, 105, 2 I I-2 I3.

Kambouris, A. A. et al. (1976). Ileal loop uretero-ileostomy in patients with neurogenic bladder. American f. Surgery, 131, 224-227.

KenEALY, J. C. (I965). Considerations in urinary diversion of paraplegic patients. $\mathcal{F}$. Urology, 94, I I 2. 
LeAdBetter, W. F. \& ShafFer, F. G. (I965). Ileal loop diversion: its application to the treatment of neurogenic bladder dysfunction. F. Urology, 75, 470-479.

MacKenzie, A. D. \& Ankenman: G. J. (I960). Urinary diversion to the isolated ileal segment. Canadian F. Surgery, 4, 43-49.

REECE, R. W. \& HACKLER, R. H. (I975). Vesico-ureteroplasty in the paraplegic: long-term follow-up in 77 patients. F. Urology, 113, 474-476.

Skinner, D. G., Gottesman, J. E., \& Richie, J. P. (I975). The isolated sigmoid segment: its value in temporary urinary diversion and reconstruction. F. Urology, 113, 614-618.

W'ALKER, W. C. \& WISE, M. F. (I969). Uretero-ileostomy in management of neurogenic bladders in the adult. F. Urology, 102, 325-329.

W'atT, R. C. (I974). Urinary diversion. American f. Nursing, I4, I806-I8I I.

Woodhead, D. M. \& Porch, P. P., JR. (I964). Cutaneous uretero-ileostomy for neurogenic vesical dysfunction. F. Urology, 91, 253-255.

WYATT, J. K. (1974). Ileal conduit diversion for benign disease: a critical review and long-term follow-up. Canadian F. Surgery, 17, 270-273. 